\title{
CICLO BIOLÓGICO Y COMPORTAMIENTO DE Udea secticastalis HAMPSON (LEPIDOPTERA: PYRALIDAE)
}

\author{
Menandro S. Ortiz ${ }^{1}$ \\ Rubén M. Pulido ${ }^{2}$
}

\begin{abstract}
RESUMEN
El presente trabajo se llevó a cabo en el insectario del Departamento de Entomología de la Universidad Nacional Agraria, durante cinco meses continuados. Se obtuvieron tres generaciones sucesivas de Udea secticastalis Hampson. Se observó las diferentes etapas de desarrollo, detallándose la duración de cada unos de ellos, comparándose con especies relacionadas de misma familia y observadas por diferentes autores. Además se incluye el comportamiento de la larva y del adulto.
\end{abstract}

Palabras claves: Udea, Pyralidae, Lepidoptera.

\section{SUMMARY}

The present paper has been deployed at the insectary of the Entomological Department of the Universidad Nacional Agraria La Molina during five continued months. Three successive generations of Udea secticastalis Hampson has been observed. Different deployment stages were noticed, detailing the duration of each one of them and compared with species of the same family and observed by different authors. Also the larvae and adult behavior has been included.

Key words: Udea, Pyralidae, Lepidoptera.

\section{INTRODUCCIÓN}

En muchos lugares del mundo existe un déficit alimenticio que cada día se agudiza más, por lo cual los gobiernos de la mayoría de países han orientado una buena parte de sus esfuerzos y economía fiscal hacía la solución de éste problema que resulta bastante complejo.

El Perú no escapa a éste problema, por lo que es necesario fomentar y tecnificar la pequeña agricultura, fundamentalmente hacía los cultivos hortícolas.

En las variadas especies hortícolas conocidas, el apio (Apium graveolens) es una de las que se encuentran entre las más importantes, tanto por el área sembrada como por sus rendimientos económicos (Semsch, 1961). En nuestro país, el apio es una de las hortalizas bastante cultivadas (Esculies, 1966), tratando de mejorar la calidad en diversos centros de investigación con la finalidad de obtener mejores rendimientos.

La planta de apio es nativa de las tierras pantanosas del norte de África; luego su cultivo se extendió desde Suiza hasta Egipto y al oriente de Asia, el Cáucaso y las montañas de la India (Semsch, op. cit.). En Estados Unidos de Norteamérica su consumo se popularizó gracias a cultivadores holandesesamericanos que habitaban próximos a Michigan.

En la terapéutica antigua era muy usado como planta medicinal, considerando al zumo con propiedades antiescorbúticas, las hojas cocidas en leche contra la afonía y el asma, la infusión de semillas era

\footnotetext{
1 Facultad de Medicina Humana y Facultad de Ciencias Biológicas, Universidad Ricardo Palma, Avenida Benavides 5440, Lima, Perú, E-mail: mop@infonegocio.net.pe

${ }^{2}$ Facultad de Ciencias Biológicas, Universidad Ricardo Palma.
} 
estomática. Antiguamente esta planta no se comía ni se usaba cruda, empleándose por mucho tiempo para sopas y guisos. Más tarde en Estados Unidos de Norteamérica e Inglaterra se consumió cruda.

La hoja y el peciolo del apio constituyen la porción de la planta que tiene valor y calidad, determinando consecuentemente su valor comercial. Del mismo modo estas estructuras de la planta presentan caracteres físicos y químicos que determinan los nutrientes del que se disponen.

Para el cultivo del apio, el clima ideal es aquel que contenga baja humedad atmosférica y bastante luminosidad; como lo es la región de la sierra de nuestro país (Esculies op. cit.). En algunas otras localidades diferentes también es posible su cultivo; pero se debe tener mucho cuidado por el ataque de plagas y enfermedades que puede tener.

Sobre éste último factor, excluyendo los factores climáticos y edáficos, se nos presenta la evidencia inobjetable de las estadísticas que nos indican la magnitud de las mermas producidas por las plagas, enfermedades y malezas.

Respecto a las plagas se hallan los lepidópteros de las familias Noctuidae y Pyralidae, hemípteros de las familias Cicadellidae y Aphididae y ácaros de la familia Tetranychidae. El éxito del control de plagas depende del conocimiento que se tenga de las especies de insectos dañinos, por lo cual el estudio de los aspectos básicos de biología, hábitos, capacidad de oviposición, porcentaje de fertilidad de los huevos, longevidad de los adultos, influencia de la temperatura y humedad sobre el desarrollo de Udea secticastalis Hampson constituye un aporte a la solución racional de éste problema con la finalidad de ir cubriendo este vacío; y que son los objetivos del presente trabajo.

\section{ANTECEDENTES}

En nuestro país no se han realizado trabajos sobre Udea secticastalis Hampson, ni tampoco existen trabajos relacionados con especies del mismo género como son $U$. profundalis (Packard) y U. rubigalis (Guenée), las que también se hospedan sobre apio, registradas en otras regiones zoogeográficas.

Sólo se cuenta con el trabajo de Wille (1952) quien cita a Nomophilla noctuella (D. et S.) en la costa central, estando más bien ésta especie distribuida en
Europa (Wolf, 1975) y que probablemente se refirió a $U$. secticastalis.

Sobre la biología de especies de éste género se encuentran pocos trabajos, destacando los de Metcalf y Flint (1965) y Tamaki y Butt (1977). Estos últimos sólo refieren que el desarrollo larval presenta 5 estadíos y es completado en 17,58 días en $U$. profundalis. Por otro lado Metcalf y Flint (op. cit.) presentan datos adicionales sobre $U$. rubigalis. Refieren que el período de incubación varía de 5 a 12 días, señalando además que la pre-pupa tiene una duración promedio de 2 días y que el estado de pupa oscila entre 10 a 12 días, según la temperatura imperante. Agregan que la duración total del ciclo de desarrollo es de aproximadamente 40 días, pudiendo haber 7 a 8 generaciones al año en los invernaderos. Finalmente señalan que ésta especie parece estar afectada adversamente por temperaturas mayores a $30^{\circ} \mathrm{C}$.

Sobre el comportamiento, Tamaki y Butt (op. cit.) refieren que las larvas de $U$. profundalis, cuando recién emergen se alimentan principalmente de los brotes, cubriendo además las hojas con hilos de seda y excrementos hasta cuando llega aproximadamente a la mitad de su desarrollo. Estiman que la larva individualmente, durante el tiempo de su desarrollo, consume alrededor de $14,67 \mathrm{~cm}^{2}$ de hoja, siendo éste consumo cerca del $79 \%$ durante el último estadío larval.

Metcalf y Flint (op. cit.) reseñan que los insectos cuando se hallan listos para empupar, usualmente forman un estuche protector, enrollando las hojas, luego interiormente tejen un capullo de seda (cocón) y finalmente se transforman al estado de pupa, emergiendo las polillas adultas entre los 10 a 12 días. Los adultos permanecen quietos en el cultivo la mayor parte del día, volando activamente durante la noche.

Finalmente Peterson (1951) señala que ésta especie es polífaga, teniendo como plantas hospedadoras al algodonero, papa, rosa, fucsia, lechuga, crisantemo, dalia, geranio, entre otras; y evidentemente el apio.

Sobre la distribución geográfica, Munroe (1967) registró por primera vez en el Perú al género Udea Guenée al describir a $U$. punoalis procedente del valle de Chuchito, aproximadamente a $4000 \mathrm{~m}$ sobre el nivel del mar, en el Departamento de Puno. Otros registros acerca de éste género lo otorga el mismo Munroe (op. cit.) describiendo a U. decoripennis 
para Chile y $U$. soratalis, $U$. coranialis y $U$. annectans para Bolivia.

\section{MATERIALES Y MÉTODOS}

El presente trabajo fue conducido en el insectario del Departamento de Entomología de la Universidad Nacional Agraria La Molina, durante 5 meses, época en se obtuvo 3 generaciones sucesivas.

El primer paso consistió en conseguir material biológico del campo, colectándose larvas y pupas de ésta polilla. Estos estados de desarrollo junto con hojas de apio se trasladaron al insectario en donde se les dispuso en frascos de boca ancha, cubriéndolas con tela sujetada con una banda elástica, a fin de obtener adultos. Obtenidos éstos se trasladaron a otros frascos similares, alimentándolos con miel de abeja diluida en agua en una proporción de 1:3. En el interior se dispuso frasquitos con agua sosteniendo ramitas de apio para la postura de huevos.

Producida la eclosión de los huevos, se tomaron 40 larvas recién emergidas, individualizadas en vasitos de plástico, previamente numerados. La observación fue diaria para cada una de las 40 larvas a fin de obtener la duración de cada uno de los diferentes estadíos. Este se determinó midiendo el diámetro transversal de la cápsula cefálica, buscándose las exuvias para confirmar la muda producida. Formada la pupa se conservó en condiciones similares hasta la emergencia del adulto.

La determinación de la capacidad de oviposición y longevidad de los adultos se efectuaron con parejas aisladas. Se consideró 10 parejas como mínimo. Se usaron adultos recién emergidos en condiciones similares a lo anteriormente descrito, efectuándose las observaciones de modo diario.

Respecto al estudio del comportamiento se realizaron sobre los estados larval y adulto. Fue necesario usar jaulas de malla y naturalmente tomando notas sobre lo que acontece al interior de ella.

\section{RESULTADOS Y DISCUSIÓN}

Los resultados sobre la duración del ciclo de desarrollo comprende el período de incubación, de desarrollo larval y período pupal, para las tres generaciones sucesivas criadas en condiciones de insectario.

\section{Período de incubación}

Los huevos eclosionaron 10 días después de la oviposición para la primera generación, 7 días para la segunda y 5 días para la tercera generación. Los datos obtenidos concuerdan ampliamente con lo observado por Metacalf y Flint (1965) quienes encontraron que los huevos incuban entre 5 a 12 días en Udea rubigalis (Guenée), plaga de importancia en cultivo de apio. Igualmente se observa la influencia de la temperatura en la sucesión de generaciones, según los estimados de Jacob y Cox (1977), pues el registro de temperatura promedio para la incubación de los huevos de la primera generación fue de $15,6^{\circ}$ $C$ en el mes de septiembre, para la segunda generación fue de $16.8^{\circ} \mathrm{C}$ en octubre y para la tercera generación fue de $19.6^{\circ} \mathrm{C}$ en el mes de diciembre.

\section{Desarrollo Larval}

Se observa con claridad las diferencias existentes en las tres generaciones. Para la primera generación la duración promedio observada fue de 19,78 días en el mes de octubre cuando la temperatura en un insectario (en ésta oportunidad cerrado) fue de $18,4^{\circ}$ C. Luego para la segunda generación en el mes de noviembre (a partir de la cual el insectario fue abierto) fue de 23,7 días con una temperatura promedio de $17,7^{\circ}$ C. Finalmente la tercera generación necesitó 19,58 días promedio, en el mes de enero con una temperatura de $21,1^{\circ} \mathrm{C}$.

Igualmente, para ésta fase la influencia de la temperatura se hace presente, dándonos a saber que la duración del estado larval se hace más corto en la estación de verano, donde la temperatura es mayor. Los resultados obtenidos por Jacob y Cox (1977) quienes condujeron la biología del pirálido Ephestia kuehniella Zeller conforman estas variantes. En todos los casos el desarrollo del estado larval requirió de 5 estadíos larvales.

Particularmente, en el quinto y último estadío larval, se observa un requerimiento mayor de días en las tres generaciones. Cabe destacar que en promedio los últimos 1,93 días, para las tres generaciones, corresponden a una etapa inactiva del quinto estadío larval, llamada pre-pupa. Tamaki y Butt (1977) en su trabajo con $U$. profundalis (Packard) encontraron que las larvas presentan 5 estadíos antes de empupar en condiciones de medio ambiente.

Con respecto a la pre-pupa, se considera dentro del desarrollo larval por producirse sin muda adicional; $y$ 
su duración es el promedio registrado por Murat (1961) e Hinostroza (1975) para el pirálido Hellula undalis Fabricius.

La disminución relativa de la duración total del desarrollo larval bajo la influencia de la temperatura progresivamente ascendente y considerando el período pre-pupal en cada generación sucesiva, es reflejo de la duración del estado larval correspondiente, ya que la duración del período de pre - pupa para las tres generaciones no varía mayormente; siendo la duración larval total correspondiente a la primera generación de 21,45 días, para la segunda generación de 25,72 días y de 21,60 días para la tercera generación. Esta duración en condiciones de insectario, está en relación con el resultado obtenido por Prabha et al. (1978), quienes encontraron una duración variable de 19,95 a 29,3 días, al efectuar estudios biológicos con Phycita infusilla Meyrick, especie que también pertenece a la familia Pyralidae.

Por otro lado, al evaluar los datos obtenidos, se constata que la duración promedio del desarrollo larval de la segunda generación es mayor debido a que la temperatura descendió, en comparación a la primera y segunda generación, donde sí hubo una mayor temperatura.

\section{Período de Pupa}

El período de pupa es semejante al de la incubación; se reduce a medida que se suceden las generaciones. Sin embargo, se puede notar un ligero aumento en la duración de la segunda generación (10,8 días) con respecto a la primera generación (9,17 días) y luego claramente una disminución en la duración de la tercera generación. Es preciso, para ello, anotar la influencia de la temperatura en éste período, tal como se notó en el desarrollo de los estados anteriores. Esta duración, con los rangos observados, son significativamente semejantes a los observados por Metcalf y Flint (1965) en $U$. rubigalis (Guenée).

\section{Ciclo Total de Desarrollo}

Udea secticastalis Hampson presenta para las condiciones de insectario, un ciclo total de desarrollo que implica una menor duración según se suceden las generaciones. Así tenemos 35,33 días para la tercera generación cuanto la temperatura fluctuaba con un promedio de $21^{\circ} \mathrm{C}$, con respecto a la primera generación (40,62 días) cuando la temperatura fue de $18,4^{\circ} \mathrm{C}$.

Igualmente, el período mínimo y máximo para completar el desarrollo total, disminuye en las nuevas generaciones, así se observa que para la primera generación el rango es de 38 a 47 días, disminuyendo en la tercera generación de 32 a 41 días.

Los experimentos demostraron por otro lado, aunque en forma no muy marcada, que las hembras completan primero el desarrollo de su ciclo total que los machos. Así mismo se menciona nuevamente que la segunda generación tiene una duración mayor (43,52 días) que la primera generación (40,62 días) dado que desarrolló bajo una menor temperatura promedio.

Los organismos sujetos normalmente a temperaturas variables en la naturaleza dentro de un rango permisible, propenden a adaptarse a éstos cambios; y por ello podría explicarse la duración del ciclo en las siguientes generaciones criadas en insectario a partir de la primera generación, cuando se elevó la temperatura.

Metcalf y Flint (1965) encontraron en U. rubigalis (Guenée) que la duración del ciclo de vida es de alrededor de 40 días. Datos relacionados encontraron Bouhelier y Hudalt (1935) y Wille (1952) para el pirálido Hellula undalis Fabricius, señalando duraciones menores en los meses de verano y otoño y períodos mayores para completar el ciclo de desarrollo durante los meses más fríos.

Así mismo Wille (op. cit.) amplía sus obervaciones para la especie tratada, indicando que el número de generaciones son 4, durante el verano y otoño; y que probablemente éstas sean 8 al año. Igualmente, Metcalf y Flint (op. cit.) señalan que en $U$. rubigalis (Guenée) pueden existir 7 ú 8 generaciones en los invernaderos. Finalmente señalan que ésta especie es adversamente afectada por temperaturas mayores a $28^{\circ} \mathrm{C}$. Hecho similar ha sido posible observar en el presente trabajo en donde generaciones posteriores no se obtuvo una población semejante como al inicio de éste ciclo biológico.

\section{Período de Pre-Oviposición}

Se considera a este período al comprendido entre la emergencia del adulto y el inicio de la oviposición. Este período tuvo una duración que varía en las tres generaciones sucesivas, sin embargo la duración mínima observada fue de 2 días para las tres generaciones. La mayor variabilidad se presenta en las generaciones anteriores (8 días máximo en la primera generación) a la tercera generación (4 días máximo); por lo tanto debido a la acción de los factores físicos medio ambientales, principalmente la temperatura, la duración promedio es menor 
conforme suceden las generaciones entendiéndose que debe haber un límite, pudiendo observarse una menor variabilidad en la tercera generación (3,5 días con respecto a la primera generación (4,1 días).

\section{Período de Oviposición}

Las hembras depositan los huevos en un período, en donde se notó una tendencia a la disminución en el número de ellos, según se suceden las generaciones. Se apreció que para la primera generación el promedio de oviposición es de 6,20 días, para la segunda generación es de 4,83 días y 3,66 para la tercera generación.

La oviposición se completa en un período mínimo de 1 a 3 días, mientras que el máximo varía de 6 (segunda y tercera generación) a 8 días (primera generación). Este período se completa en la mayor parte de las hembras varios días antes de su muerte.

\section{Período de Post-Oviposición}

Se considera al período comprendido entre el término de la oviposición y la muerte del adulto. Durante éste período las hembras presentaron una duración no similar a los períodos precedentes (pre-oviposición y oviposición). Sino más bien de modo irregular. Así la duración de éste período para la segunda generación fue de 3,0 días y 4,16 días para la tercera generación, menores con respecto a la primera generación que posee un tramo de 6,20 días, debido probablemente a que es una generación cuyos padres proceden del campo. En cambio las dos últimas generaciones proceden de una crianza llevada a cabo en insectario, por lo que sus valores son más próximos.

\section{Longevidad en Relación al Sexo}

Considerando sólo adultos apareados, la longevidad por sexo en promedio varió notablemente en cada generación, manteniéndose las hembras vivas por mayor tiempo. La longevidad máxima para machos (9,8 días) y hembras (15,5 días) se observó en la primera generación; mientras que la longevidad mínima para machos (8 días) y de hembras $(10,4$ días) se observaron en la última generación obtenida en el presente trabajo.

\section{Longevidad de Adultos no Apareados}

Considerando adultos machos no apareados, la longevidad promedio por generación no fue muy variable, manteniéndose más bien casi estable; pero sí resultaron más longevos que los adultos apareados.

Los adultos hembras no apareadas presentaron una longevidad promedio casi constante por generación, es decir, presentaron un rango estrecho de variabilidad. Esta longevidad con respecto a la de los adultos hembras apareadas, resultó ser similar.

Comparando longevidad entre machos y hembras no apareados resultaron con mayor longevidad éstas últimas, de modo similar que el caso de adultos apareados.. El promedio obtenido para machos no apareados fue de 10,2 días y para hembras no apareadas fue de 12,5 días.

\section{Capacidad y Ritmo de Oviposición}

La capacidad de oviposición de cada hembra es muy variable. Se encontró que las hembras ovipositaron de 3 hasta 414 huevos, con un promedio de 167,5 huevos, teniendo en cuenta a las tres generaciones observadas.

El número máximo de huevos se observó en la primera generación; éste resultó ser muy superior al promedio. Girlind (1978) encontró un potencial de oviposición entre 400 a 600 huevos por hembra en un trabajo realizado con Eldana saccharina (Walker). Prabha et al.(1978) encontraron que Phycita infusilla Meyrick pone casi 100 huevos por hembra.

Como se deducirá, en los Pyralidae, que agrupa a las especies antes citadas, al igual que las de Udea, parece ser que el número de huevos por hembra es variable; presentando nuestra especie en estudio una oviposición que se le puede considerar relativamente alta.

La cantidad de huevos ovipositados por las hembras, disminuye en promedio conforme suceden las generaciones; así ésta reducción se observó en la tercera generación (114,83 huevos) con respecto a la segunda generación (221 huevos).

El ritmo de oviposición diario en las diferentes generaciones en general, presentó una tendencia similar en todas ellas, depositando el mayor número de huevos los primeros días hasta el cuarto o quinto día; y luego ésta cantidad disminuye progresivamente hasta ser nulo entre el séptimo y noveno días. El máximo número de huevos se observó al tercer día.

El mayor número de huevos por día se dio en la primera generación, disminuyendo luego dichas cantidades en las sucesivas generaciones. En la tercera generación, si bien hubo poca oviposición, ésta luego se levantó proporcionalmente, pero en menor número que en generaciones anteriores. 


\section{Relación de la Longevidad de la Hembra y el Número de Huevos}

Observando el promedio de huevos puestos por hembra y su longevidad también promedio, de cada generación, se aprecia que existe una relación directamente proporcional entre éstas dos variables, es decir que a mayor longevidad la capacidad de oviposición es también mayor. Este hecho se deduce destacando el máximo promedio de huevos puestos (221 huevos) por la máxima longevidad promedio de hembra (15,5 días) que se dio en la primera generación; mientras que el promedio mínimo de huevos puestos (114,83 huevos) por una longevidad promedio mínima de hembras se dio en la tercera generación.

\section{Fertilidad de Hembras}

De las tres generaciones estudiadas, en la segunda se observó un $25 \%$ de infertilidad, correspondiendo un $100 \%$ de fertilidad potencial a las otras generaciones. Sin embargo no todas las hembras que ovipositaron dieron lugar a huevos viables, observándose en cada generación una marcada tendencia a disminuir la viabilidad de huevos.

Así, de la primera a la tercera generación, el porcentaje de hembras que pusieron huevos no viables fue de 20, 50 y 83,3\%, respectivamente; es decir que las hembras que ovipositaron y cuyos huevos fueron viables decreció hasta $16,7 \%$ en la tercera generación. Este hecho pueda deberse probablemente a una serie de factores como por ejemplo que las hembras no copularon o no fueron receptivas o copularon con machos estériles o puede haber existido una cópula imperfecta. Por otro lado, debe tenerse en cuenta que la baja de fertilidad de las hembras por generación, pudo verse afectada adversamente por la temperatura cada vez mayor, tal como los señala Metcalf y Flint (1965). Así mismo debe tenerse en cuenta la influencia que ejerce la luz; ya que ésta especie parece ser un insecto de días cortos.

\section{Proporción de sexos}

Se aprecia que la producción de hembras en individuos criados en insectario fue ligeramente mayor al de los machos, aunque esto no sucedió en la segunda generación, pues aquí la proporción de sexos fue de 1:1. Por lo tanto se estima que en el campo ésta proporción estaría equilibrada lo cual asegura la sucesión de generaciones con poblaciones apreciables. Estos resultados obtenidos están acordes con los que presentaron El-Kilf et al. (1974) con Palpita unionalis Hübner, también polilla de la familia Pyralidae.

\section{Comportamiento}

Al eclosionar los huevos, las larvitas emergen dirigiéndose a los brotes, donde inician el daño a las hojas, según el lugar de oviposición. Cuando atacan a los brotes, las hojitas son reunidas mediante hilos de seda para construir un lugar protegido. En tal situación consumen totalmente a los brotes atacados. Cuando se dirigen a las hojas se posesionan en el envés del limbo, plegando los bordes de ésta o con las hojas vecinas mediante los hilos de seda ya citados, alimentándose dentro de éstos túneles. A la hoja la consumen hasta llegar a la nervadura central.

Una larva puede destruir más de una planta pequeña e igualmente, ésta puede albergar más de una larva en sus primeros estadíos de desarrollo. En plantas más desarrolladas, las larvas de los dos primeros estadíos y aún del tercero, raspan las hojas. En el cuarto estadío larval ya se hace notorio que la larva come la hoja, practicándole perforaciones.

En todos los casos el lugar donde las larvas se posesionan, se va a encontrar abundante excrementos de las mismas, de apariencia granular y húmeda, ensuciando las hojas. Estos coprolitos les dan una característica típica al daño ocasionado, además de los hilos de seda plegando a las hojas o brotes, según lo citado anteriormente.

La larva de quinto estadío sigue siendo muy voraz, con abundante coprolitos; plega más a las hojas y empieza a formar un capullo de hilos de seda en el que permanecen hasta dar lugar a la pupa.

Los adultos emergen rompiendo la cubierta pupal por la parte antero-dorsal. Una vez fuera el nuevo adulto mueve las antenas y camina inmediatamente por un corto trecho después del cual permanece quieto. En éste momento el color no es muy nítido, pero se puede diferenciar los colores y ornamentación que presenta el ala del adulto maduro, aunque éstas aún no han sido extendidas.

Luego de casi una hora se observa que el adulto está apto para volar, después que las alas se han extendido, dejando al descubierto el dorso del abdomen. También se observó la expulsión de un líquido marrón rojizo por el ano, quedando finalmente el nuevo adulto completamente hábil.

Al iniciar el vuelo, es característica la forma zigzageante pudiendo observarse también un vuelo alto y sostenido; durante el día permanecen escondidos entre las hojas, especialmente en el envés. Estos adultos son de hábitos nocturnos tal como lo 
señalan Bohulier y Hudault (1935) y Metcalf y Flint (1965).

La cópula se realiza en la noche, estimándose sea en la noche del primer día. Durante éste acto el macho y la hembra permanecen unidos en sentido opuesto por un determinado tiempo (cuando se intentó determinar el tiempo, estas polillas interrumpían la cópula, por la aproximación de una luz muy tenue).

Las hembras ovipositan durante la noche, las posturas se realizan generalmente en el envés de las hojas, aunque eventualmente pueden hacerlo en el haz y en toda la hoja. Esto fue observado por Wille (1952), quien mencionó además que el peciolo servía también como lugar de oviposición. Los huevos son puestos en grupos superpuestos, constituyendo masas y a veces de manera individual. Esta forma de oviposición concuerda con lo citado por Metcalf y Flint (1965) y Wille (1952).

\section{CONCLUSIONES}

- El período de incubación disminuye conforme se sucede las generaciones, correspondiendo para cada una de éstas: 10, 7 y 5 días respectivamente.

- $\quad$ Presenta 5 estadíos larvales, cuya duración de cada una de ellas es variable en las diferntes generaciones obtenidas, siendo el último estadío larval el que requirió mayor tiempo para su desarrollo.

- El tiempo requerido para el completo desarrollo larval en las tres generaciones, son: 21,45; 25,72 y 21,60 días, respectivamente.

- La larva tiene por hábito el pegar las hojas, ubicándose dentro de ésta cubierta protectora, donde se alimenta y posteriormente empupa, protegiéndose éste con hilos de seda, formando un capullo (cocón).

- Las hembras apareadas tienen una longevidad promedio para las tres generaciones de 12,21 días, similar al de las hembras no apareadas. En ambos casos resultan ser más longevas que los machos.

- Los machos apareados muestran una menor longevidad que los machos no apareados.

- El potencial de reproducción en condiciones de invernadero disminuye en $65,5 \%$ en la tercera generación.

- La máxima oviposición se produce al tercer día de iniciado este período.

- $\quad$ La proporción de sexos es 1:1.

\section{LITERATURA CITADA}

BOUHELIER \& Hudault. 1935. Note Sur Hellula undalis F., Pyrale aux cruciferes dans le Maroc occidental. Rev. Path Veg., 22: 123-130.

EL-KIFL, A.H., A.L. Andel - Salam \& A.M.M. Rahhal. 1974. Biological studies of the olive leafmoth, Palpita unionalis Hb. (Lep.L Pyralidae). Bull. Soc. Entomol. Egypte 58: 337334.

HINOSTROZA, F.H. 1975. Biología y comportamiento del «gusano del brote de la col» Hellula undalis F. (Lep.: Pyralidae). Tesis para optar el título de Ingeniero Agrónomo. Universidad Nacional Agraria La Molina, Lima-Perú, 98 pp.

ESCULIES, O. 1966. Ensayo del uso de herbicidas químicos en el cultivo del apio. Tesis para optar el título de Ingeniero Agrónomo. Universidad Nacional Agraria La Molina, Lima-Perú, 78 pp.

GIRLIND, D.J. 1978. The distribution and biology of Eldana saccharina Walker (Lep.: Pyralidae). Bull. Entomol. Res. 68 (3): 471-488.

JACOB, T.A. \& P.D. Cox. 1977. The influence of temperature an humidity on the life - cycle of Ephestia kuehniella Zeller (Lep.: Pyralidae). J. Stored Prod. Res. 13 (3): 107-118.

METCALF, C.L. \& W.P. Flint. 1965. Insectos destructivos e insectos útiles, sus costumbres y su control. Cia. Ed. Contiental. S. A. Mexico, 761, 979-981

MUNROE, E. 1967. Tour new species of Udea from South America (Lep.: Pyralidae). Can. Ent. 99 (4): 3992-394.

MURAT, I. 1961. El gusano perforador del nabo chino criollo (Raphanus sativus Major) y de la col (Brassica oleracea var. Capitata) Hellula undalis Fab. y su control químico. Tesis para optar el título de Ingeniero Agrónomo. Universidad Nacional Agraria La Molina, Lima-Perú, 87 pp.

PETERSON, A. 1951. Larvae of insects. An introduction to neartic species. Part I: Lepideoptera and Plant infesting Hymenoptera. Columbus-Ohio, $315 \mathrm{pp}$.

PRABHA, S., M.S. Dhinsa, A.K Dhawan \& S.S. Dhillon. 1978. Biology of the cotton bud moth, Phycita infusella Meyr. (Lep.: Pyralidae). J. Entomol. Res. 2 (1): 33-39

SEMSCH, C. 1961. Ensayo del uso de diferentes dosis de nitrógeno y fósforo en el cultivo del apio. Tesis para optar el título de Ingeniero Agrónomo. Universidad Nacional Agraria La Molina, LimaPerú, 83 pp. 
TAMAKI, G. \& B.A. Butt. 1977. Biology of the nfalse celery leaftier and damage to sugarbeets. Environ. Entomol. 6(1): 35-38.

WOLFF, N.L. 1975. The Nomophila species (Lep.; Pyralidae) found in the North Atlantic area. Entomol. Medd. 43 (3): 129-135.
WILLE, J. E. 1952. Entomología Agrícola del Perú. Segunda Ed. Dir. Agr., Min. Agr., Lima, Perú, 543 pp. 